\title{
Design of three-dimensional visualization indoor navigation system in smart city construction
}

\author{
Chunlei Guo ${ }^{1}$, Cuizhi $\mathrm{Liu}^{2}$, and Erhu Wei ${ }^{3, *}$ \\ ${ }^{1}$ Department of Civil Engineering, Shenyang Urban Construction College, Shenyang, Liaoning, 110167, China \\ ${ }^{2}$ Department of Surveying and Mapping Engineering, College of Resources and Civil Engineering, North eastern University, Shenyang, \\ Liaoning, 110819, China \\ ${ }^{3}$ School of Geodesy and Geomatics, Wuhan University, Wuhan, Hubei, 430079, China
}

\begin{abstract}
In recent years, smart city has attracted more and more attention because of its high intelligence and informatization. In the future, smart community, smart parking lot and smart exhibition hall will enter people's life. In these smart services, indoor navigation and positioning technology is the basic technology. High precision indoor and outdoor seamless positioning technology plays a vital role in people's future life, is the science and technology source power to promote public innovation and entrepreneurship, and is an important part of supporting the national strategic needs. At present, indoor navigation is still facing challenges such as high cost, poor adaptability and poor endurance. In this paper, combined with threedimensional(3D) visualization technology, indoor navigation is designed and applied. The results show that the indoor navigation system combines indoor positioning and artificial intelligence to achieve multichannel positioning and reduce power consumption, which can be widely used in the construction of smart city and bring convenience to people's life.
\end{abstract}

\section{Introduction}

In the 21st century, with the rapid development of wireless communication technology and Internet technology, people's demand for location-based services is growing, so wireless navigation and positioning technology has made great progress. ${ }^{[1]}$ So far, GPS, Beidou and other satellite positioning systems have been able to provide all-weather, real-time navigation services. Although the positioning of these satellite systems is fast and accurate, the signal can not penetrate the heavy concrete to provide positioning and navigation for indoor space.

Compared with outdoor, due to the complexity of indoor environment and the privacy of indoor data, the digitization of indoor space is difficult and the data accuracy is low. ${ }^{[2]}$ So far, $90 \%$ of indoor data are still offline. It is more and more urgent to digitize indoor environment and provide location-based services with indoor data. In the indoor environment, due to the occlusion of buildings, the satellite signal decays rapidly, and the effective position information can not be obtained from it, resulting in poor positioning effect. With the increasing demand for indoor positioning, it is urgent to develop various technologies suitable for indoor positioning and navigation according to the data obtained from indoor digitization. ${ }^{[3]}$

Many scholars at home and abroad have studied the methods and technologies of indoor navigation and 3D visualization under the construction of smart city: The
University of Cambridge in the UK has proposed the infrared based active badge indoor positioning system, apple in the US has developed the ibeacon indoor positioning system based on wireless local area networks (WLAN), Microsoft has developed the radar indoor positioning system based on received signal strength indication (RSSI) Hong Kong University of science and technology has proposed the LANDMARC positioning system based on radio frequency identification (RFID), and Beijing University of Posts and telecommunications has proposed the "deer hunting" indoor positioning system. The research and development of indoor navigation and positioning technology is changing with each passing day, but the application of this technology is still in constant exploration. ${ }^{[4]}$ In the future, the development of indoor navigation and positioning technology with high accuracy, low cost and universality and the realization of indoor and outdoor seamless navigation and positioning are always the hot spots and difficulties at home and abroad. ${ }^{[5]}$

The purpose of this study is to use the mobile 3D scanning system to digitize the indoor space, import the results of digitization (mainly point cloud data and panoramic image) into the platform of indoorviewer, and use the platform to publish the $3 \mathrm{D}$ real scene information of indoor space online for the use of terminal equipment.

\footnotetext{
Corresponding author: ehwei@sgg.whu.edu.cn

Corresponding address: School of Geodesy and Geomatics, Wuhan University, 430079
} 


\section{Construction idea of 3D visualization indoor navigation system}

The platform integrates three-dimensional panoramic roaming, point of interest editing and adding, path planning, two-dimensional and three-dimensional length and area measurement and other functions, and provides a third-party API development interface to meet the customized needs of customers. ${ }^{[6]}$

As a mobile terminal, the navigation app, through docking platform, enables users to use their mobile phones to accurately locate their position in the indoor space. ${ }^{[7]}$ At the same time, relying on the powerful path planning ability of the platform, users can select the destination in the indoor space, conduct indoor navigation and reach the destination.

\section{Overall design of 3D visualization indoor navigation system}

\section{1 overall logical architecture}

(1) Through the mobile app to achieve three-dimensional remote live preview;

(2) Convenient background data management and map package timely update;

(3) Edit and update the navigation path in time;

(4) Text, picture, audio and video information display of interest points;

(5) IOT sensor connection, real-time monitoring.

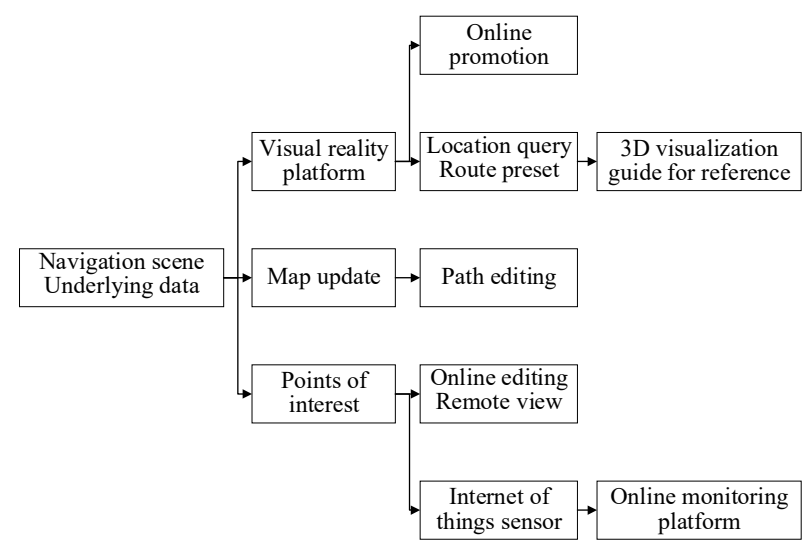

Fig. 1. Overall design framework

\section{2 client system architecture}

Based on the construction of a virtual 3D visualization scene map of building space, the following function modules are realized: route navigation (same floor, cross floor), remote path planning, mobile phone scanning for route map, $3 \mathrm{D}$ visualization roaming and so on.

\section{3 technical implementation plan}

\subsection{1 preliminary preparation}

According to the needs of customers and the scale of indoor space, make preliminary analysis of the project, communicate with each other, confirm the technical requirements of the project, and formulate a reasonable implementation plan. Before the start of the project, customers need to provide the plan of indoor space, so as to define the data acquisition area, divide the data acquisition sub area, calculate the time required for data acquisition, and plan the preliminary data acquisition route. Before the indoor shooting, it is necessary to initiate the shooting application process with the relevant departments in advance: send the application letter (explaining the background and purpose of coming), so as to facilitate the property to coordinate with the relevant enterprises in advance, and select the appropriate time for data collection on the premise of not affecting the public interest.

\subsection{2 field exploration}

Before the start of data acquisition, it is necessary to carry out field survey on the site, modify the planned data acquisition route according to the site conditions and the specific conditions of measurement, and mark the change plan on the map. If there is no ready-made plan for the survey area, the general plane structure of the survey area can be drawn and the data collection route can be marked at the same time of field survey and route planning.

\subsection{3 data acquisition}

After arriving at the scene, according to the planned route, combined with the actual situation, the data collection of point cloud and panoramic photos is carried out.

\subsection{4 data processing and improvement}

After collecting the data, the professional software is used to process and generate the point cloud and panoramic photo results. At the same time, the results are published on the platform of indoorviewer, and the functions of 3D panoramic roaming, VR house viewing, 2D house type map navigation, path planning, and point of interest interaction are realized in the platform.

The automatically processed data in the instance of indexviewer needs to be improved and edited later to ensure the smooth deployment of the map and navigation scheme.

\subsection{5 navigation map generation}

(1) Generation of 2D plan

The post-processing software of the system will automatically generate two-dimensional plane quality map to check the integrity of data acquisition and the 
whole process. In the indexviewer, full color twodimensional plane map can be automatically generated according to the point cloud data.

(2) Perfecting the automatically generated navigation map in the indoorviewer

You can associate different floors by editing and linking related navigation nodes, such as stairs or elevators. The wrong navigation node and association will lead to incorrect path planning and need to be deleted. Test different path planning several times to confirm the correctness of path calculation and navigation logic.

\subsection{Function of 3D visualization indoor navigation system}

\subsection{1 indoor space quickly obtain positioning information and plan the optimal path}

Users can browse the indoor space remotely through the platform or official app to quickly and effectively find the elevator, shop, service desk, etc.

At the same time, it can also provide users with facilities navigation of internal environment, vehicle and route information query, etc. in the points of interest to realize the visual guidance of action.

Support cross floor, indoor and outdoor integrated navigation to meet the needs of customers' positioning and navigation.
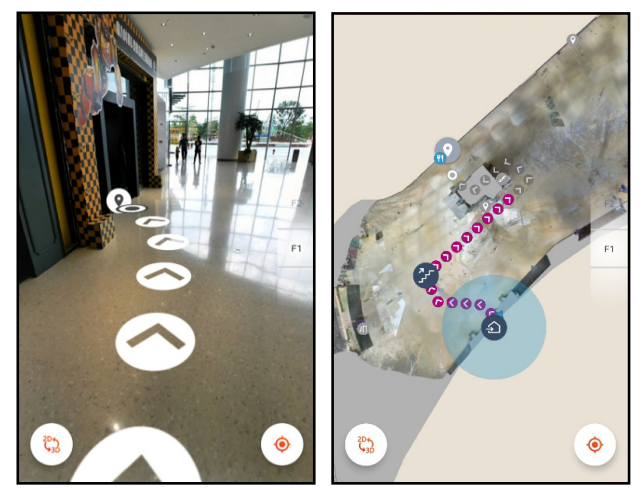

Fig. 2. Route planning

\subsection{2 indoor positioning and navigation can be realized without GPS signal}

Based on three-dimensional map and positioning navigation, it realizes the safety assurance and intelligent management of people, vehicles and objects in the parking lot, and creates an intelligent, convenient and safe intelligent shopping environment.
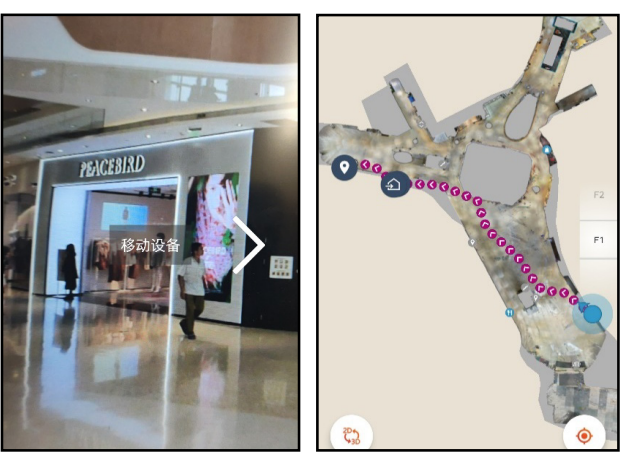

Fig. 3. Positioning and navigation

\subsection{3 $2 D$ and $3 D$ guide to online virtual reality scene}

VR visual effect, can switch between 2D plan and 3D panoramic view at will.
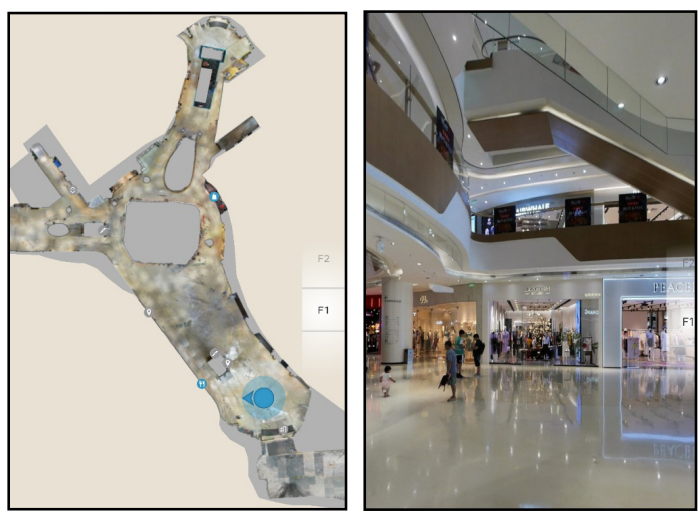

Fig. 4. 2D and 3D visual effect

\subsection{4 parking module}

Through the development, parking information query, reservation and parking navigation can be realized. Parking mark, reverse search, pick-up, settlement integration function support.

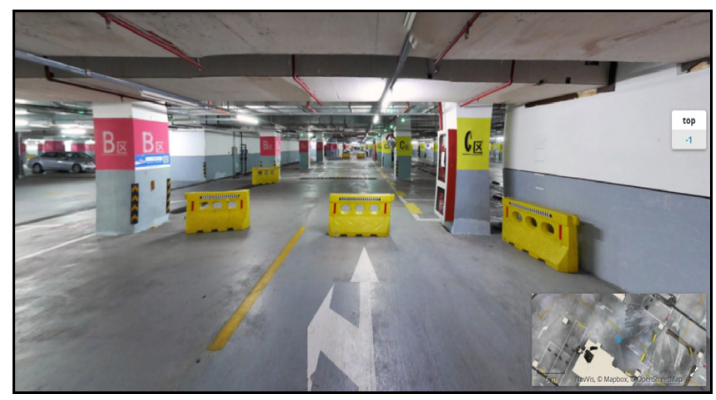

Fig. 5. Parking module

\subsection{5 link sharing}

In order to facilitate users to share their own location, the function of one click sharing specific location information is developed. If users can't determine their location in the indoor space, they can share the link with their friends and view the shared location after opening it. At the same time, this function can also be used to share the preview of the location of the store. After opening 
the link, you can view the real scene of the current location.

\subsection{6 information query}

The system has built-in query bar, which is convenient for shopping malls, transportation hubs and other facilities to query and maximize user experience.

\subsection{7 personalized navigation}

Personalized customized navigation menu bar, one click to open, the background of the system will classify all kinds of facilities, convenient for users to search on demand.
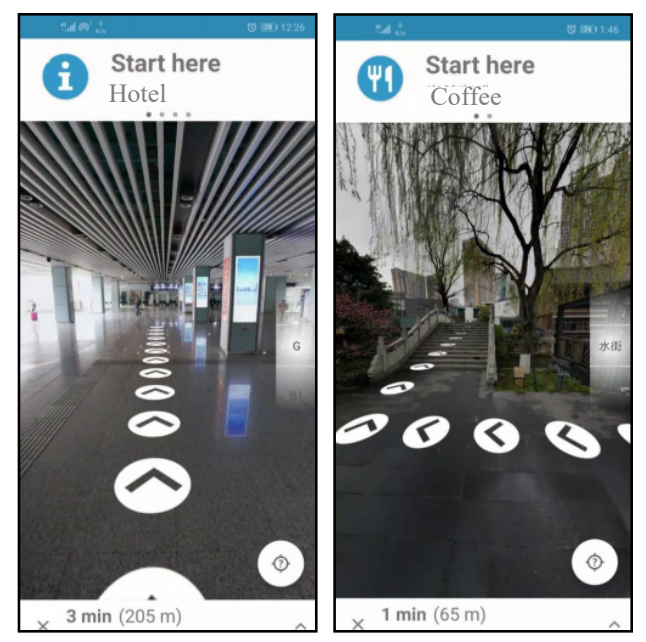

Fig. 6. Personalized Guide

\subsection{8 online purchase}

Add points of interest to the goods and enter the price information to complete one click process services such as query, appointment, payment, pick-up and online purchase based on the store information.

\subsection{9 information display}

In the system, there is the point cloud data of the mall with location information. You can add the points of interest of goods or shops with additional location information in the point cloud. For display and publicity.

\subsubsection{0 data update}

When the layout of the mall or the appearance of the facilities changes greatly, contact the development team to re scan and post process the mall data in a short time for application. It maximizes the shortening of construction period and minimizes the decline of user experience caused by layout change.

\section{Conclusions}

At present, indoor navigation still faces many challenges, mainly including: 1) high cost and complexity, such as infrared, ultrasonic and other hardware facilities are required to achieve positioning and navigation, which limits the popularity of indoor positioning; 2) It has poor adaptability to dynamic environment, and the movement of some people or objects will affect the positioning accuracy; 3) The endurance is poor. For $\mathrm{Wi} \mathrm{Fi}$, Bluetooth, vision, inertial navigation and other positioning technologies that can be integrated into smart phones, the battery consumption is very fast when the positioning is turned on, and the positioning cannot be continued for a long time. 4) It is vulnerable to multipath interference in NLOS; 5) The positioning accuracy of complex indoor scene is relatively low; 6) Poor scalability, such as some technologies such as infrared, Bluetooth, etc. are only suitable for short distance, and can not do anything for long distance.

With the continuous development of indoor positioning, it is believed that these deficiencies will be improved in the future. Therefore, we look forward to the development trend of indoor positioning in the construction of smart city in the future: 1) multi sensor combined positioning will be one of the important directions for the development of indoor precise positioning in the future; 2) Low power consumption of equipment is realized; 3) It is compatible, easy to integrate and expand; 4) Reduce the cost and technical threshold; 5) Precise integration algorithm for moving people and objects; 6) Indoor positioning and artificial intelligence combined development. In the future, indoor positioning will shine brilliantly in all areas of people's life and bring convenience to our life.

\section{Acknowledgments}

This paper is one of the phased achievements of national key R \& D Program (2018YFC1503600) and National Natural Science Foundation of China (41874036).

\section{References}

1. Liu, GX, Shi, LF. (2018)Overview of indoor navigation and positioning technology. Journal of navigation and positioning, 6 (2): 7-14.

2. Gao, W, Hou, CY, Xu, WM. et al. (2019) Research progress and Prospect of indoor navigation and positioning technology. Journal of navigation and positioning, 7 (1): 10-17.

3. Li, JL. (2015) Research on hand-held 3D scanning technology without mark points based on grating projection. Harbin Institute of technology, Harbin.

4. Chen, RZ, Chen, L. (2017) Development status and challenges of indoor positioning technology based on smart phones. Acta Surveying \& mapping, 46 (10): 1316-1326.

5. He, YR, Zheng, YM, Pan, HP. et al. (2016) True 3D modeling and application of complex buildings 
based on point cloud data. Remote sensing technology and application, 31 (6): 1091-1099.

6. Wei, HT. (2018) Error modelling for multi-sensor measurements in infrastructure-free indoor navigation. Sersors, 18(2): 590-595.

7. KRAGIC D. (2019) Object seach and localization for an indoor mobile robot. Journal of Computing \& Information Technology,17(1): 67-80. 